Submitted to the Annals of Applied Probability

arXiv: arXiv:0000.0000

\title{
PSEUDO-VALUE APPROACH FOR CONDITIONAL QUANTILE RESIDUAL LIFETIME ANALYSIS FOR CLUSTERED SURVIVAL AND COMPETING RISKS DATA WITH APPLICATION TO BONE MARROW TRANSPLANT DATA - SUPPLEMENTARY MATERIALS
}

\author{
By Kwang Woo Ahn and Brent R. Logan \\ Medical College of Wisconsin
}

\begin{abstract}
This supplementary material includes the proofs of (3.1) and (3.2) of Section 3, the asymptotic property of $\hat{S}(t \mid \mathbf{Z})$, and the convergence of $P_{i j}\left(\hat{Q}_{\tau}+t_{0}\right)$. In addition, we illustrate the proposed method for the survival setting.
\end{abstract}

1. Proof of (3.1) and (3.2). We prove (3.1) and (3.2) of Section 2 by following the arguments of Logan, Zhang and Klein (2011). We consider the competing risks setting to show (3.1). The proof of (3.2) can be similarly done. We have

$$
\begin{aligned}
P_{i j}^{f}(t)= & n \hat{F}_{1}(t)-(n-1) \hat{F}_{1}^{-i j}(t) \\
= & \sum_{a=1}^{m} \sum_{b=1}^{\ell} \frac{N_{1 a b}(t)}{\hat{G}\left(X_{a b}\right)}-\sum_{(a, b) \neq(i, j)} \frac{N_{1 a b}(t)}{\hat{G}^{-i j}\left(X_{a b}\right)} \\
= & \frac{N_{1 i j}(t)}{G\left(X_{i j}\right)}+\sum_{(a, b) \neq(i, j)} N_{1 a b}(t)\left[\left\{\frac{1}{\hat{G}\left(X_{a b}\right)}-\frac{1}{G\left(X_{a b}\right)}\right\}\right. \\
& \left.-\left\{\frac{1}{\hat{G}^{-i j}\left(X_{a b}\right)}-\frac{1}{G\left(X_{a b}\right)}\right\}\right]+N_{1 i j}(t)\left\{\frac{1}{\hat{G}\left(X_{i j}\right)}-\frac{1}{G\left(X_{i j}\right)}\right\} .
\end{aligned}
$$

The third term of (1.1) $N_{1 i j}(t)\left\{1 / \hat{G}\left(X_{i j}\right)-1 / G\left(X_{i j}\right)\right\}$ is $O_{p}\left(m^{-1 / 2}\right)$ due to the consistency of $\hat{G}(t)$. Consider the second term of (1.1). Let

$$
\begin{aligned}
R(t) & =\sum_{a=1}^{m} \sum_{b=1}^{\ell} I\left(X_{a b} \geq t\right), M^{c}(t)=\sum_{a=1}^{m} \sum_{b=1}^{\ell} M_{a b}^{c}(t), \\
M^{c(-i j)}(t) & =\sum_{(a, b) \neq(i, j)} \sum_{a b}^{c}(t) .
\end{aligned}
$$

Using

$$
\frac{1}{\hat{G}\left(X_{a b}\right)}-\frac{1}{G\left(X_{a b}\right)}=\frac{1}{\hat{G}\left(X_{a b}\right)} \int_{0}^{X_{a b}} \frac{\hat{G}(u-)}{G(u) R(u)} d M^{c}(u),
$$

Keywords and phrases: pseudo-value, residual lifetime, clustered data 
the second term of (1.1) is equal to

$$
\begin{aligned}
& \sum_{(a, b) \neq(i, j)} \sum_{1 a b}(t)\left\{\frac{1}{\hat{G}\left(X_{a b}\right)} \int_{0}^{X_{a b}} \frac{\hat{G}(u-)}{G(u) R(u)} d M^{c}(u)\right. \\
& \left.-\frac{1}{\hat{G}^{-i j}\left(X_{a b}\right)} \int_{0}^{X_{a b}} \frac{\hat{G}^{-i j}(u-)}{G(u) R(u)} d M^{c(-i j)}(u)\right\} .
\end{aligned}
$$

Thus, the second term of (1.1) becomes

$$
\begin{aligned}
& \sum_{(a, b) \neq(i, j)} \sum_{\hat{G}\left(X_{a b}\right) \hat{G}^{-i j}\left(X_{a b}\right)} \\
& \quad \times \int_{0}^{X_{a b}}\left\{\frac{N^{-i j}\left(X_{a b}\right) \hat{G}(u-)}{G(u) R(u)}-\frac{\hat{G}\left(X_{a b}\right) \hat{G}^{-i j}(u-)}{G(u) R^{-i j}(u)}\right\} d M^{c(-i j)}(u) \\
& \quad+\sum_{(a, b) \neq(i, j)} \frac{N_{1 a b}(t)}{\hat{G}\left(X_{a b}\right)} \int_{0}^{X_{a b}} \frac{\hat{G}(u-)}{G(u) R(u)} d M_{i j}^{c}(u) .
\end{aligned}
$$

Consider the first term of (1.2). It can be seen that

$$
\begin{aligned}
& \frac{n(n-1)}{\sqrt{m}} \int_{0}^{X_{a b}}\left\{\frac{\hat{G}^{-i j}\left(X_{a b}\right) \hat{G}(u-)}{G(u) R(u)}-\frac{\hat{G}\left(X_{a b}\right) \hat{G}^{-i j}(u-)}{G(u) R^{-i j}(u)}\right\} d M^{c(-i j)}(u) \\
& =\int_{0}^{X_{a b}}\left\{\frac{(n-1) \hat{G}^{-i j}\left(X_{a b}\right) \hat{G}(u-)}{G(u) R(u) / n}-\frac{n \hat{G}\left(X_{a b}\right) \hat{G}^{-i j}(u-)}{G(u) R^{-i j}(u) /(n-1)}\right\} \frac{d M^{c(-i j)}(u)}{\sqrt{m}}
\end{aligned}
$$

converges in distribution to some random variable $K_{a b}$. Furthermore, because $\hat{G}(t) \rightarrow_{p} G(t)$ uniformly by Zhou et al. (2012), the first term of (1.2) is asymptotically equivalent to

$$
\begin{aligned}
& \frac{\sqrt{m}}{n} \sum_{(a, b) \neq(i, j)} \sum_{(n-1) \hat{G}\left(X_{a b}\right) \hat{G}^{-i j}\left(X_{a b}\right)} \\
& =\frac{\sqrt{m}}{n(n-1)} \sum_{(a, b) \neq(i, j)} \frac{N_{1 a b}(t) K_{a b}(t)}{G\left(X_{a b}\right)^{2}}+O_{p}\left(m^{-1 / 2}\right) \text {. }
\end{aligned}
$$

Since $\left(N_{1 a b}(t) K_{a b}(t) / G\left(X_{a b}\right)^{2} ; b=1, \ldots, \ell\right)^{T}$ 's are iid and $n=m \times \ell$, the first term of $(1.2)$ is $O_{p}\left(m^{-1 / 2}\right)$ by multivariate central limit theorem. We can show that the second term of (1.2) is asymptotically equivalent to

$$
\sum_{(a, b) \neq(i, j)} \frac{N_{1 a b}(t)}{G\left(X_{a b}\right)}\left[\int_{0}^{X_{a b}} \frac{1}{R(u)} d M_{i j}^{c}(u)\right] .
$$

Using the definition of $M_{i j}^{c}(t)$, we have

$$
\int_{0}^{X_{a b}} \frac{1}{R(u)} d M_{i j}^{c}(u)=\int_{0}^{X_{i j}} \frac{I\left(u \leq X_{a b}\right)}{R(u)} d M_{i j}^{c}(u) .
$$

imsart-aap ver. 2014/10/16 file: "Paper final - Supplemental".tex date: March 28, 2016 
Thus, we have

$$
\begin{aligned}
& \sum_{(a, b) \neq(i, j)} \frac{N_{1 a b}(t)}{G\left(X_{a b}\right)}\left[\int_{0}^{X_{a b}} \frac{1}{R(u)} d M_{i j}^{c}(u)\right] \\
& =\int_{0}^{X_{i j}} \frac{1}{R(u) / n} \frac{1}{n} \sum_{(a, b) \neq(i, j)} \frac{N_{1 a b}(t) I\left(X_{a b} \geq u\right)}{G\left(X_{a b}\right)} d M_{i j}^{c}(u) .
\end{aligned}
$$

Consider

$$
\frac{1}{n} \sum_{(a, b) \neq(i, j)} \frac{N_{1 a b}(t) I\left(X_{a b} \geq u\right)}{G\left(X_{a b}\right)}
$$

By the law of large numbers, (1.3) converges in probability to

$$
E\left\{\frac{N_{1 a b}(t) I\left(X_{a b} \geq u\right)}{G\left(X_{a b}\right)}\right\}
$$

We have

$$
\begin{aligned}
& E\left\{\frac{N_{1 a b}(t) I\left(X_{a b} \geq u\right)}{G\left(X_{a b}\right)}\right\} \\
= & E\left[E\left\{\frac{I\left(T_{a b} \leq t\right) I\left(\epsilon_{a b}=1\right) I\left(T_{a b} \leq C_{a b}\right) I\left(X_{a b} \geq u\right)}{G\left(X_{a b}\right)} \mid T_{a b}\right\}\right] \\
= & E\left[E\left\{\frac{I\left(T_{a b} \leq t\right) I\left(\epsilon_{a b}=1\right) I\left(T_{a b} \leq C_{a b}\right) I\left(T_{a b} \geq u\right)}{G\left(T_{a b}\right)} \mid T_{a b}\right\}\right] \\
= & E\left[\frac{I\left(T_{a b} \leq t\right) I\left(\epsilon_{a b}=1\right) I\left(T_{a b} \geq u\right)}{G\left(T_{a b}\right)} E\left\{I\left(T_{a b} \leq C_{a b}\right) \mid T_{a b}\right\}\right] \\
= & E\left\{I\left(T_{a b} \leq t\right) I\left(\epsilon_{a b}=1\right) I\left(T_{a b} \geq u\right)\right\} \\
= & P\left(u \leq T_{f} \leq t, \epsilon=1\right) .
\end{aligned}
$$

Note that $R(t) / n$ converges to $P\left(T_{f} \geq u\right) P(C \geq u)=P\left(T_{f} \geq u\right) G(u)$. Then, the third term of (1.1) is asymptotically equivalent to

$$
\int_{0}^{X_{i j}} \frac{P\left(T_{f} \leq t, \epsilon=1 \mid T_{f} \geq u\right)}{G(u)} d M_{i j}^{c}(u)
$$

which completes the proof of (3.1) of Section 3 .

2. Asymptotics of $\hat{\boldsymbol{S}}(\boldsymbol{t} \mid \mathbf{Z})$. In this section, we study the asymptotic distribution of $\hat{S}(t \mid \mathbf{Z})$. Let $M_{i j}(t)=N_{i j}(t)-\int_{0}^{t} I\left(X_{i j} \geq u\right) \lambda_{0}(u) \exp \left(\boldsymbol{\beta}^{T} \mathbf{Z}_{i j}(u)\right)$. 
Assume that $T_{i j} \in[0, \mathcal{T}]$. Define

$$
\begin{aligned}
\Xi^{(r)}(\boldsymbol{\beta}, t) & =\frac{1}{m} \sum_{i=1}^{m} \sum_{j=1}^{\ell} I\left(X_{i j} \geq t\right) \exp \left\{\boldsymbol{\beta}^{T} \mathbf{Z}_{i j}(t)\right\} \mathbf{Z}_{i j}(t)^{\otimes r} \\
\xi^{(r)}(\boldsymbol{\beta}, t) & =E\left\{\Xi^{(r)}(\boldsymbol{\beta}, t)\right\} \\
\mathcal{E}(\boldsymbol{\beta}, t) & =\frac{\Xi^{(1)}(\boldsymbol{\beta}, t)}{\Xi^{(0)}(\boldsymbol{\beta}, t)}, \mathbf{e}(\boldsymbol{\beta}, t)=\frac{\xi^{(1)}(\boldsymbol{\beta}, t)}{\xi^{(0)}(\boldsymbol{\beta}, t)} \\
\mathcal{V}(\boldsymbol{\beta}, t) & =\frac{\Xi^{(2)}(\boldsymbol{\beta}, t)}{\Xi^{(0)}(\boldsymbol{\beta}, t)}-\mathcal{E}(\boldsymbol{\beta}, t)^{\otimes 2}, v(\boldsymbol{\beta}, t)=\frac{\xi^{(2)}(\boldsymbol{\beta}, t)}{\xi^{(0)}(\boldsymbol{\beta}, t)}-\mathbf{e}(\boldsymbol{\beta}, t)^{\otimes 2} \\
\mathbf{U}(\boldsymbol{\beta}) & =\sum_{i=1}^{m} \sum_{j=1}^{\ell} \int_{0}^{\mathcal{T}}\left\{\mathbf{Z}_{i j}(u)-\mathbf{e}(\boldsymbol{\beta}, u)\right\} d M_{i j}(u) \\
\mathcal{I}(\boldsymbol{\beta}) & =\sum_{i=1}^{m} \sum_{j=1}^{\ell} \int_{0}^{\mathcal{T}} \mathcal{V}(\boldsymbol{\beta}, u) d N_{i j}(u) \\
\mathbf{w}_{i j} & =\int_{0}^{\mathcal{T}}\left\{\mathbf{Z}_{i j}(u)-\mathbf{e}(\boldsymbol{\beta}, u)\right\} d M_{i j}(u)
\end{aligned}
$$

Under the regularity assumptions, Spiekerman and Lin (1998) and Lee, Wei and Amato (1992) showed that $\sqrt{m}(\hat{\boldsymbol{\beta}}-\boldsymbol{\beta})$ is asymptotically equivalent to $\mathbf{A}^{-1} m^{-1 / 2} \mathbf{U}(\boldsymbol{\beta})$, where $\mathcal{I}\left(\boldsymbol{\beta}^{*}\right)$ converges in probability to $\mathbf{A}$ for $\boldsymbol{\beta}^{*}$ on the line segment between $\boldsymbol{\beta}$ and $\hat{\boldsymbol{\beta}}$. For the details of the regularity assumptions, see Spiekerman and Lin (1998). Furthermore, Spiekerman and Lin (1998) showed that $\sqrt{m}\{\hat{\Lambda}(t)-\Lambda(t)\}=m^{-1 / 2} \sum_{i=1}^{m} \Psi_{i}(t)+o_{p}(1)$, where

$$
\Psi_{i}(t)=\int_{0}^{t} \frac{d M_{i \cdot}(u)}{\xi^{(0)}(\boldsymbol{\beta}, t)}-\left\{\int_{0}^{t} e(\boldsymbol{\beta}, u) \lambda_{0}(u) d u\right\}^{T} \mathbf{A}^{-1} \mathbf{w}_{i \cdot .}
$$

Using the delta method, $\sqrt{m}\{\hat{S}(t \mid \mathbf{Z})-S(t \mid \mathbf{Z})\}$ is asymptotically equivalent to

$$
\begin{aligned}
& -\sqrt{m} S(t \mid \mathbf{Z})\left\{\hat{\Lambda}(t) \exp \left(\hat{\boldsymbol{\beta}}^{T} \mathbf{Z}\right)-\Lambda(t) \exp \left(\boldsymbol{\beta}^{T} \mathbf{Z}\right)\right\} \\
& =-\sqrt{m} S(t \mid \mathbf{Z})\left[\left\{\hat{\Lambda}(t) \exp \left(\hat{\boldsymbol{\beta}}^{T} \mathbf{Z}\right)-\hat{\Lambda}(t) \exp \left(\boldsymbol{\beta}^{T} \mathbf{Z}\right)\right\}\right. \\
& \left.+\left\{\hat{\Lambda}(t) \exp \left(\boldsymbol{\beta}^{T} \mathbf{Z}\right)-\Lambda(t) \exp \left(\boldsymbol{\beta}^{T} \mathbf{Z}\right)\right\}\right] \\
& =-\sqrt{m} S(t \mid \mathbf{Z})\left[\hat{\Lambda}(t) \exp \left(\hat{\boldsymbol{\beta}}^{T} \mathbf{Z}\right) \mathbf{Z}^{T}(\hat{\boldsymbol{\beta}}-\boldsymbol{\beta})\right. \\
& \left.+\exp \left(\boldsymbol{\beta}^{T} \mathbf{Z}\right)\{\hat{\Lambda}(t)-\Lambda(t)\}\right]+o_{p}(\sqrt{m}) .
\end{aligned}
$$

imsart-aap ver. 2014/10/16 file: "Paper final - Supplemental".tex date: March 28, 2016 
Therefore, $-\hat{S}(t \mid \mathbf{Z})+S(t \mid \mathbf{Z})$ is asymptotically equivalent to

$$
\begin{aligned}
& S(t \mid \mathbf{Z})\left\{\Lambda(t) \exp \left(\boldsymbol{\beta}^{T} \mathbf{Z}\right) \mathbf{Z}^{T} \mathbf{A}^{-1} \frac{1}{m} \sum_{i=1}^{m} \sum_{j=1}^{\ell} \int_{o}^{\mathcal{T}}\left\{\mathbf{Z}_{i j}(u)-\mathbf{e}(\boldsymbol{\beta}, u)\right\} d M_{i j}(u)\right. \\
& \left.+\exp \left(\boldsymbol{\beta}^{T} \mathbf{Z}\right) \frac{1}{m} \sum_{i=1}^{m} \Psi_{i}(t)\right\} \\
& =\frac{1}{m} \sum_{i=1}^{m}\left\{\boldsymbol{\phi}_{i}(t)+S(t \mid \mathbf{Z}) \exp \left(\boldsymbol{\beta}^{T} \mathbf{Z}\right) \Psi_{i}(t)\right\},
\end{aligned}
$$

where

$$
\phi_{i}(t)=S(t \mid \mathbf{Z}) \Lambda(t) \exp \left(\boldsymbol{\beta}^{T} \mathbf{Z}\right) \mathbf{Z}^{T} \mathbf{A}^{-1} \sum_{j=1}^{\ell} \int_{o}^{\mathcal{T}}\left\{\mathbf{Z}_{i j}(u)-\mathbf{e}(\boldsymbol{\beta}, u)\right\} d M_{i j}(u) .
$$

Thus, $\sqrt{m}\{\hat{S}(t \mid \mathbf{Z})-S(t \mid \mathbf{Z})\}$ is asymptotically equivalent to a sum of $m$ iid random variables. By the multivariate central limit theorem, $\sqrt{m}\{\hat{S}(t \mid \mathbf{Z})-$ $S(t \mid \mathbf{Z})\}$ follows a normal distribution with mean 0 and variance $E\left\{\phi_{i}(t)+\right.$ $\left.S(t \mid \mathbf{Z}) \exp \left(\boldsymbol{\beta}^{T} \mathbf{Z}\right) \Psi_{i}(t)\right\}^{2}$.

3. Proof of convergence of $P_{i j}\left(\hat{Q}_{\tau}+t_{0}\right)$. Using

$$
P_{i j}^{f}(t)=\frac{N_{1 i j}(t)}{G\left(X_{i j}\right)}+\int_{0}^{X_{i j}} \frac{P\left(T_{f} \leq t, \epsilon=1 \mid T_{f} \geq u\right)}{G(u)} d M_{i j}^{c}(u)+O_{p}\left(m^{-1 / 2}\right),
$$

$P_{i j}^{f}\left(\hat{Q}_{\tau}+t_{0}\right)$ is asymptotically equivalent to

$$
\frac{N_{1 i j}\left(\hat{Q}_{\tau}+t_{0}\right)}{G\left(X_{i j}\right)}+\int_{0}^{X_{i j}} \frac{P\left(T_{f} \leq \hat{Q}_{\tau}+t_{0}, \epsilon=1 \mid T_{f} \geq u\right)}{G(u)} d M_{i j}^{c}(u) .
$$

Because $N_{1 i j}(x)$ is continuous at $x=Q_{\tau}+t_{0}$ with probability one and $\hat{Q}_{\tau}$ is consistent, the first term converges in probability to

$$
\frac{N_{1 i j}\left(Q_{\tau}+t_{0}\right)}{G\left(X_{i j}\right)}
$$

Because of the continuity of $F_{1}(t)$ and consistency of $\hat{Q}_{\tau}$, the second term converges to

$$
\int_{0}^{X_{i j}} \frac{P\left(T_{f} \leq Q_{\tau}+t_{0}, \epsilon=1 \mid T_{f} \geq u\right)}{G(u)} d M_{i j}^{c}(u),
$$

which completes the proof.

imsart-aap ver. 2014/10/16 file: "Paper final - Supplemental".tex date: March 28, 2016 
4. Survival setting. In this section, we propose a test to compare the conditional quantile residual lifetimes of multiple groups for independent and clustered survival data. The marginal Cox proportional hazards regression (Lee, Wei and Amato, 1992) is used to model the marginal hazard function of $T_{i j}$, which is

$$
\lambda\left(t \mid \mathbf{Z}_{i j}\right)=\exp \left(\boldsymbol{\beta}^{T} \mathbf{Z}_{i j}\right) \lambda_{0}(t),
$$

where $\lambda_{0}(t)$ is an arbitrary baseline hazard function. Under the independence working assumption, $\boldsymbol{\beta}$ is estimated by minimizing the partial likelihood

$$
\prod_{i=1}^{m} \prod_{j=1}^{\ell}\left\{\frac{\exp \left(\hat{\boldsymbol{\beta}}^{T} \mathbf{Z}_{a b}\right)}{\sum_{a=1}^{m} \sum_{b=1}^{\ell} I\left(X_{a b} \leq X_{i j}\right) \exp \left(\hat{\boldsymbol{\beta}}^{T} \mathbf{Z}_{a b}\right)}\right\} .
$$

The Breslow estimator for $\Lambda_{0}(t)$ is estimated by

$$
\hat{\Lambda}_{0}(t)=\sum_{i=1}^{m} \sum_{j=1}^{\ell} \frac{I\left(X_{i j} \leq t\right) \Delta_{i j}}{\sum_{a=1}^{m} \sum_{b=1}^{\ell} I\left(X_{a b} \leq X_{i j}\right) \exp \left(\hat{\boldsymbol{\beta}}^{T} \mathbf{Z}_{a b}\right)} .
$$

Lee, Wei and Amato (1992) and Spiekerman and Lin (1998) studied the consistency of $\hat{\boldsymbol{\beta}}$ and $\hat{\Lambda}_{0}(t)$, and their asymptotic properties for clustered survival data. The conditional survival probability at time $t$ can be estimated by

$$
\hat{S}(t \mid \mathbf{Z})=\exp \left\{-\int_{0}^{t} \exp \left(\hat{\boldsymbol{\beta}}^{T} \mathbf{Z}\right) \hat{\Lambda}_{0}(d u)\right\} .
$$

Note that $\hat{S}(t \mid \mathbf{Z})$ is asymptotically normal with convergence rate $\sqrt{m}$ given $t$. Let $q_{\tau}$ be the conditional $\tau$ th quantile residual lifetime survival to time $t_{0}$ given $\mathbf{Z}=\mathbf{z}_{0}$. To estimate $q_{\tau}$ we consider

$$
\begin{aligned}
& U\left(q_{\tau}\right)=S\left(t_{0}+q_{\tau} \mid \mathbf{z}_{0}\right)-(1-\tau) S\left(t_{0} \mid \mathbf{z}_{0}\right)=0 \\
& \hat{U}\left(q_{\tau}\right)=\hat{S}\left(t_{0}+q_{\tau} \mid \mathbf{z}_{0}\right)-(1-\tau) \hat{S}\left(t_{0} \mid \mathbf{z}_{0}\right)=0 .
\end{aligned}
$$

We assume that $S\left(t \mid \mathbf{z}_{0}\right)$ is absolutely continuous and $f(t)=d S\left(t \mid \mathbf{z}_{0}\right) / d t$ is positive on some neighborhood of $q_{\tau}+t_{0}$. Then, $U\left(q_{\tau}\right)$ has a unique solution. In addition, $\hat{U}\left(q_{\tau}\right)$ converges to $U\left(q_{\tau}\right)$ because $\hat{S}\left(t \mid \mathbf{z}_{0}\right)$ is consistent. In practice, $\hat{q}_{\tau}$ can be uniquely determined by defining it as the smallest $q$ at which $\hat{S}\left(t_{0}+q_{\tau} \mid \mathbf{z}_{0}\right)-(1-\tau) \hat{S}\left(t_{0} \mid \mathbf{z}_{0}\right)$ crosses 0 . Therefore, $\hat{q}_{\tau}$ is consistent given $\tau$.

Assume that we compare the $\tau$ th conditional quantile residual lifetime given survival to time $t$ among $\zeta$ groups when $\mathbf{Z}=\mathbf{z}_{0}$. Under the null hypothesis, $q_{1 \tau}=\cdots=q_{\zeta \tau} \equiv q_{0 \tau}$, where $q_{i \tau}$ is the $\tau$ th conditional quantile residual lifetime given survival to time $t$ of group $i$ when $\mathbf{Z}=\mathbf{z}_{0}$. Let $S_{g}\left(t \mid \mathbf{z}_{0}\right)$

imsart-aap ver. 2014/10/16 file: "Paper final - Supplemental".tex date: March 28, 2016 
be a survival probability at time $t$ given $\mathbf{Z}=\mathbf{z}_{0}$ for group $g$. Define $U_{g}\left(q_{\tau}\right)=$ $S_{g}\left(t_{0}+q_{\tau} \mid \mathbf{z}_{0}\right)-(1-\tau) S_{g}\left(t_{0} \mid \mathbf{z}_{0}\right)$ for $g=1, \ldots, \zeta$. Under the null hypothesis, testing $q_{1 \tau}=\cdots=q_{\zeta \tau} \equiv q_{0 \tau}$ is equivalent to testing $U_{1}\left(q_{0 \tau}\right)=\cdots=$ $U_{\zeta}\left(q_{0 \tau}\right)=0$, which is tested by GEE treating pseudo-values as a response variable. Under the null hypothesis, $q_{0 \tau}$ can be consistently estimated by solving $\hat{S}\left(t_{0}+q_{\tau} \mid \mathbf{z}_{0}\right)-(1-\tau) \hat{S}\left(t_{0} \mid \mathbf{z}_{0}\right)=0$ based on the pooled data. To test if $U_{1}\left(q_{0 \tau}\right)=\cdots=U_{\zeta}\left(q_{0 \tau}\right)=0$, we obtain pseudo-values $P_{i j}^{s}\left(t_{0}\right)$ and $P_{i j}^{s}\left(t_{0}+\right.$ $\left.\hat{q}_{0 \tau}\right)$ for $i=1, \ldots, m$ and $j=1, \ldots, \ell$. Note that $P_{i j}^{s}\left(t_{0}\right)$ and $P_{a b}^{s}\left(t_{0}+q_{\tau}\right)$ are asymptotically independent when $i \neq a$. Assuming $N_{i j}$ is continuous at $t_{0}+q_{0 \tau}$ with probability one, it can be shown that $P_{i j}^{s}\left(t_{0}+\hat{q}_{0 \tau}\right)$ converges in probability to $P_{i j}^{s}\left(t_{0}+q_{0 \tau}\right)$.

We parameterize a joint model using a link function $h(\cdot)$ so that

$$
\begin{aligned}
\mu_{i j}^{s}\left(t_{0}+\hat{q}_{\tau}\right)=S_{g}\left(t_{0}+\hat{q}_{\tau} \mid \mathbf{Z}_{i j}\right) & =h^{-1}\left(\phi+\phi_{1}+\gamma_{1}^{T} \mathbf{Z}_{i j}+\theta_{1 g}\right), \\
\mu_{i j}^{s}\left(t_{0}\right)=S_{g}\left(t_{0} \mid \mathbf{Z}_{i j}\right) & =h^{-1}\left(\phi+\gamma_{2}^{T} \mathbf{Z}_{i j}+\theta_{2 g}\right),
\end{aligned}
$$

where $\theta_{1 \zeta}=\theta_{2 \zeta}=0$. Here $\phi$ and $\phi+\phi_{1}$ are intercept terms for $h\left\{\mu_{i j}^{s}\left(t_{0}\right)\right\}$ and $h\left\{\mu_{i j}^{s}\left(t_{0}+\hat{q}_{\tau}\right)\right\}$, respectively, and $\theta_{1 g}$ and $\theta_{2 g}$ are parameters for an indicator function of group $g=1, \ldots, \zeta-1$ for $h\left\{\mu_{i j}^{s}\left(t_{0}+\hat{q}_{\tau}\right)\right\}$ and $h\left\{\mu_{i j}^{s}\left(t_{0}\right)\right\}$, respectively. In matrix notation, consider the response variable vector $\mathbf{P}_{i}=$ $\left\{\mathbf{P}_{i}^{s}\left(t_{0}+\hat{q}_{\tau}\right), \mathbf{P}_{i}^{s}\left(t_{0}\right)\right\}^{T}$ for $i=1, \ldots, m$. The explanatory variable defined by $\boldsymbol{\alpha}=\left(\phi, \phi_{1}, \gamma_{1}^{T}, \boldsymbol{\theta}_{1}^{T}, \boldsymbol{\gamma}_{2}^{T}, \boldsymbol{\theta}_{2}^{T}\right)^{T}$, where $\boldsymbol{\theta}_{k}=\left(\theta_{k 1}, \ldots, \theta_{k \zeta-1}\right)^{T}$ for $k=1,2$. Define the mean vector as $\boldsymbol{\mu}_{i}=\left\{\boldsymbol{\mu}_{i}^{s}\left(t_{0}+\hat{q}_{\tau}\right), \boldsymbol{\mu}_{i}^{s}\left(t_{0}\right)\right\}^{T}$ for $i=1, \ldots, m$. Then, the GEE is defined as follows:

$$
\sum_{i}\left(\frac{\partial \boldsymbol{\mu}_{i}}{\partial \boldsymbol{\alpha}}\right)^{\prime} \mathbf{V}_{i}^{-1}\left(\mathbf{P}_{i}^{s}-\boldsymbol{\mu}_{i}\right) \equiv \sum_{i} \mathbf{A}_{i}(\boldsymbol{\alpha})=\mathbf{0} .
$$

Then, $\sqrt{m}(\hat{\boldsymbol{\alpha}}-\boldsymbol{\alpha})$ converges in distribution to $N(\mathbf{0}, \Sigma)$ (Liang and Zeger, 1986). The sandwich estimator is used to estimate $\Sigma_{s}$ as follows:

$$
\hat{\Sigma}_{s}=\mathcal{I}(\hat{\boldsymbol{\alpha}})^{-1} \widehat{\operatorname{Var}}\{\mathbf{A}(\hat{\boldsymbol{\alpha}})\} \mathcal{I}(\hat{\boldsymbol{\alpha}})^{-1},
$$

where

$$
\mathcal{I}(\boldsymbol{\alpha})=\sum_{i}\left(\frac{\partial \boldsymbol{\mu}_{i}}{\partial \boldsymbol{\alpha}}\right)^{\prime}\left(\mathbf{V}_{i}\right)^{-1}\left(\frac{\partial \boldsymbol{\mu}_{i}}{\partial \boldsymbol{\alpha}}\right), \widehat{\operatorname{Var}}\{\mathbf{A}(\hat{\boldsymbol{\alpha}})\}=\sum_{i} \mathbf{A}_{i}(\hat{\boldsymbol{\alpha}}) \mathbf{A}_{i}(\hat{\boldsymbol{\alpha}})^{\prime} .
$$

Under the null hypothesis, we can estimate $U_{g}\left(\hat{q}_{0 \tau}\right)$ with $\hat{\boldsymbol{\alpha}}$ from (4.1) by

$$
\begin{aligned}
\hat{U}_{1}\left(\hat{q}_{0 \tau}\right)= & h^{-1}\left(\hat{\phi}+\hat{\phi}_{1}+\hat{\gamma}_{1}^{T} \mathbf{z}_{0}+\hat{\theta}_{1 g}\right)-(1-\tau) h^{-1}\left(\hat{\phi}+\hat{\gamma}_{2}^{T} \mathbf{z}_{0}+\hat{\theta}_{2 g}\right), \\
& \text { if } g=1, \ldots, \zeta-1 ; \\
\hat{U}_{\zeta}\left(\hat{q}_{0 \tau}\right)= & h^{-1}\left(\hat{\phi}+\hat{\phi}_{1}+\hat{\gamma}_{1}^{T} \mathbf{z}_{0}\right)-(1-\tau) h^{-1}\left(\hat{\phi}+\hat{\gamma}_{2}^{T} \mathbf{z}_{0}\right), \text { if } g=\zeta .
\end{aligned}
$$


Using the delta method, we can show that under the null hypothesis $\left\{\hat{U}_{1}\left(\hat{q}_{0 \tau}\right), \ldots, \hat{U}_{\zeta}\left(\hat{q}_{0 \tau}\right)\right\}^{T}$ converges in distribution to $N\left(\mathbf{0}, \Omega_{s} \Sigma_{s} \Omega_{s}^{T}\right)$, where

$$
\Omega_{s}=\left(\begin{array}{cccc}
\frac{\partial U_{1}\left(\hat{q}_{0 \tau}\right)}{\partial \phi} & \frac{\partial U_{1}\left(\hat{q}_{0 \tau}\right)}{\partial \phi_{1}} & \cdots & \frac{\partial U_{1}\left(\hat{q}_{0 \tau}\right)}{\partial \theta_{2 \zeta}-1} \\
\frac{\partial U_{2}\left(\hat{q}_{0 \tau}\right)}{\partial \phi} & \frac{\partial U_{2}\left(\hat{q}_{0 \tau}\right)}{\partial \phi_{1}} & \cdots & \frac{\partial U_{2}\left(\hat{q}_{0 \tau}\right)}{\partial \theta_{2 \zeta-1}} \\
\vdots & \vdots & \ddots & \vdots \\
\frac{\partial U_{\zeta}\left(\hat{q}_{0 \tau}\right)}{\partial \phi} & \frac{\partial U_{\zeta}\left(\hat{q}_{0 \tau}\right)}{\partial \phi_{1}} & \cdots & \frac{\partial U_{\zeta}\left(\hat{q}_{0 \tau}\right)}{\partial \theta_{2 \zeta-1}}
\end{array}\right) .
$$

We can obtain $\hat{\Omega}_{s}$ by plugging $\hat{\boldsymbol{\alpha}}^{s}$ into $\Omega_{s}$. Let

$$
\bar{U}\left(\hat{q}_{0 \tau}\right)=\frac{1}{n} \sum_{g=1}^{\zeta} n_{g} \hat{U}_{g}\left(\hat{q}_{0 \tau}\right) .
$$

Define $\mathbf{I}_{\zeta}$ as a $\zeta \times \zeta$ identity matrix and $\mathbf{1}_{\zeta}$ is a $\zeta \times 1$ vector consisting of 1 . Then, under the null hypothesis $\left\{\hat{U}_{1}\left(\hat{q}_{0 \tau}\right)-\bar{U}\left(\hat{q}_{0 \tau}\right), \ldots, \hat{U}_{\zeta}\left(\hat{q}_{0 \tau}\right)-\bar{U}\left(\hat{q}_{0 \tau}\right)\right\}^{T}$ converges in distribution to $N\left(\mathbf{0}, \mathbf{B} \Omega_{s} \Sigma_{s} \Omega_{s}^{T} \mathbf{B}^{T}\right)$, where $\mathbf{B}$ is defined as in the competing risks setting. Note that $\mathbf{B} \Omega_{s} \Sigma_{s} \Omega_{s}^{T} \mathbf{B}^{T}$ is not invertible. To test if $U_{1}\left(q_{0 \tau}\right)=\cdots=U_{\zeta}\left(q_{0 \tau}\right)$, we propose a test statistic

$$
\begin{aligned}
X^{2}= & m\left\{\hat{U}_{1}\left(\hat{q}_{0 \tau}\right)-\bar{U}\left(\hat{q}_{0 \tau}\right), \ldots, \hat{U}_{\zeta}\left(\hat{q}_{0 \tau}\right)-\bar{U}\left(\hat{q}_{0 \tau}\right)\right\}^{T}\left(\mathbf{B} \hat{\Omega}_{s} \hat{\Sigma}_{s} \hat{\Omega}_{s}^{T} \mathbf{B}^{T}\right)^{-} \\
& \times\left\{\hat{U}_{1}\left(\hat{q}_{0 \tau}\right)-\bar{U}\left(\hat{q}_{0 \tau}\right), \ldots, \hat{U}_{\zeta}\left(\hat{q}_{0 \tau}\right)-\bar{U}\left(\hat{q}_{0 \tau}\right)\right\}
\end{aligned}
$$

where $\left(\mathbf{B} \hat{\Omega}_{s} \hat{\Sigma}_{s} \hat{\Omega}_{s}^{T} \mathbf{B}^{T}\right)^{-}$is a generalized inverse of $\mathbf{B} \hat{\Omega}_{s} \hat{\Sigma}_{s} \hat{\Omega}_{s}^{T} \mathbf{B}^{T}$. Under the null hypothesis, $X^{2}$ converges in distribution to chi-squared distribution with degrees of freedom $\zeta-1$. By relying on GEE, we can account for not only the correlation within cluster, but also the correlation between $\hat{S}_{g}\left(t_{0}+\hat{q}_{\tau} \mid \mathbf{z}_{0}\right)$ and $\hat{S}_{g}\left(t_{0} \mid \mathbf{z}_{0}\right)$ for $g=1, \ldots, \zeta$. For one-sample test, let

$$
\omega_{s}=\left\{\frac{\partial U_{1}\left(q_{0 \tau}\right)}{\partial \phi}, \frac{\partial U_{1}\left(q_{0 \tau}\right)}{\partial \phi_{1}}, \cdots, \frac{\partial U_{1}\left(q_{0 \tau}\right)}{\partial \theta_{2 \zeta-1}}\right\}^{T} .
$$

Let $\hat{\omega}_{s}$ be an estimator of $\omega_{s}$ by plugging $\hat{\boldsymbol{\alpha}}$ into $\omega_{s}$. To test $q_{\tau}=q_{0 \tau}$, we use $m \hat{U}_{1}\left(q_{0 \tau}\right)^{2} / \hat{\mathcal{D}}$, where $\hat{\mathcal{D}}=\hat{\omega}_{s}^{T} \hat{\Sigma}_{s} \hat{\omega}_{s}$. Under the null hypothesis, $m \hat{U}_{1}\left(q_{0 \tau}\right)^{2} / \hat{\mathcal{D}}$ follows a chi-squared distribution with degree of freedom 1.

\section{References.}

Lee, E. W., Wei, L. J. and Amato, D. A. (1992). Cox-Type Regression Analysis for Large Numbers of Small Groups of Correlated Failure Time Observations in Klein, J. P. and Goel, P. K. (eds) Survival Analysis: State of the Art. Kluwer Academic Publishers, Dordrecht, Netherlands.

LiAnG, K. Y. and Zeger, S. L. (1986). Longitudinal data analysis using generalized linear models. Biometrika 73 13-22.

imsart-aap ver. 2014/10/16 file: "Paper final - Supplemental".tex date: March 28, 2016 
Logan, B., Zhang, M.-J. and Klein, J. P. (2011). Marginal models for clustered time to event data with competing risks using pseudovalues. Biometrics 67 1-7.

Spiekerman, C. F. and Lin, D. Y. (1998). Marginal regression models for multivariate failure time data. Journal of the American Statistical Association 93 1164-1175.

Zhou, B., Fine, J., Latouche, A. and Labopin, M. (2012). Competing risks regression for clustered data. Biostatistics 13 371-383.

Division of Biostatistics

Medical College of Wisconsin

E-MAIL: kwooahn@mcw.edu

blogan@mcw.edu 